\title{
Ciudadanos de la comunidad. Appropriation de la bureaucratie et expérience de la " communauté » chez les Matsigenka (Amazonie péruvienne)
}

Ciudadanos de la comunidad. Appropriation of bureaucracy and experience of "community" among the Matsigenka (Peruvian Amazon)

Ciudadanos de la comunidad. Apropiación de burocracia y experiencia de la "comunidad" entre los Matsigenka (Amazonía peruana)

\section{Raphaël Colliaux}

\section{(2) OpenEdition \\ Journals}

Édition électronique

URL : https://journals.openedition.org/jsa/17135

DOI : 10.4000/jsa. 17135

ISSN : $1957-7842$

Éditeur

Société des américanistes

Édition imprimée

Date de publication : 20 décembre 2019

Pagination : 69-90

ISSN : 0037-9174

Référence électronique

Raphaël Colliaux, «Ciudadanos de la comunidad. Appropriation de la bureaucratie et expérience de la « communauté » chez les Matsigenka (Amazonie péruvienne) », Journal de la Société des américanistes [En ligne], 105-2 | 2019, mis en ligne le 20 décembre 2019, consulté le 03 septembre 2022. URL : http://journals.openedition.org/jsa/17135; DOI : https://doi.org/10.4000/jsa.17135 


\title{
Ciudadanos de la comunidad. Appropriation de la bureaucratie et expérience de la "communauté » chez les Matsigenka (Amazonie péruvienne)
}

\author{
Raphaël ColLiauX *
}

Cet article étudie la façon dont certains membres de l'ethnie matsigenka, une population arawak du sud-est de l'Amazonie péruvienne, s'approprient et font vivre l'unité administrative au sein de laquelle ils se trouvent aujourd'hui regroupés, c'est-à-dire celle de la « communauté autochtone » (comunidad nativa). C'est le processus de formation de Palotoa-Teparo, petite communauté administrative matsigenka du département du Madre de Dios, qui sera en particulier étudié. On verra comment les Matsigenka ainsi regroupés en «communauté » reprennent à leur compte le répertoire administratif imposé par l'État, combien ils s'approprient son lexique et ses outils de manière à construire une représentativité politique qui leur est propre. De la sorte, ce sont des ramifications de l'État qu'ils ne cessent de reproduire, tout en exerçant, mutatis mutandis, une forme d'autonomie politique sur le territoire qui leur a été reconnu - aussi exigu soit-il. [Mots-clés: communauté administrative, assemblée, bureaucratie, Matsigenka, Amazonie, Pérou.]

Ciudadanos de la comunidad. Appropriation of bureaucracy and experience of "community" among the Matsigenka (Peruvian Amazon). This article examines how some members of the Matsigenka ethnic group, an Arawak population from the south-east of the Peruvian Amazon, take ownership of and relate to the administrative unit in which they are now grouped together: the comunidad nativa ("indigenous community"). Our study will focus on the formation process of Palotoa-Teparo, a small matsigenka administrative community of the Madre de Dios department. We will see how the Matsigenka thus regrouped in a "community" take over the administrative directory imposed by the state, how they appropriate its lexicon and tools in order to build a political representation of their own. They are thereby constantly reproducing ramifications of the state, all while exercising, mutatis mutandis, political sovereignty of sorts over the territory which has been alloted to them - albeit in a cramped form. [Key words: administrative community, assembly, bureaucracy, Matsigenka, Amazonia, Peru.]

Ciudadanos de la comunidad. Apropiación de burocracia y experiencia de la "comunidad" entre los Matsigenka (Amazonía peruana). Este artículo examina

* Institut français d'études andines (IFEA), Lima, Pérou [colliaux.raph@gmail.com]. 
la forma por la cual ciertos miembros del grupo étnico matsigenka, una población Arawak del sureste de la Amazonía peruana, se apropian y hacen vivir a la unidad administrativa en la que ahora están agrupados, es decir la "comunidad indígena" (comunidad nativa). Es el proceso de formación de Palotoa-Teparo, una pequeña comunidad administrativa matsigenka del departamento de Madre de Dios, que será especialmente estudiado. Veremos cómo los Matsigenka así agrupados en "comunidad" hacen suyos el repertorio administrativo impuesto por el Estado, cómo se apropian su léxico y sus herramientas para construir una representación política propia. De esta manera, son ramificaciones del Estado que reproducen constantemente, mientras ejercen, mutatis mutandis, una forma de autonomía política en el territorio que les ha sido reconocida -tan frágil como es. [Palabras clave: comunidad administrativa, asamblea, burocracia, Matsigenka, Amazonia, Perú.]

Introduit dans les basses terres en 1974, le régime de la comunidad nativa ${ }^{1}$ (communauté autochtone) a permis aux Amérindiens d'acquérir des droits fonciers collectifs, mécanisme indispensable à la défense de leur souveraineté territoriale. Toutefois, on ne peut manquer d'observer une certaine continuité historique, tant sur le plan spatial qu'idéologique, entre, d'une part, ce dispositif récemment généralisé en Amazonie et, d'autre part, les comunidades de réduction imposées dans les Andes dès les débuts de la colonie, institutions elles-mêmes organisées sur le modèle des communautés rurales de Castille, d'Estrémadure et du Royaume de Léon (Fioravanti-Molinié 1978; Zagalsky 2009). Il s'agissait, pour les colons du Xvi ${ }^{e}$ siècle, de regrouper les Amérindiens autour des principaux centres miniers et agricoles et de leur imposer subrepticement une « conception européenne de l'espace prévalant à la Renaissance, qui tendait à privilégier des structures concentrées et bien délimitées, avec, pour chaque collectivité, leurs diverses fonctions centrales, aux dépens d'autres, diffuses et dispersées, considérées comme impropres à la vie en société » (García Martínez, cité et traduit dans Herzog 2007, p. 510). Une telle généalogie explique sans nul doute l'ambiguité dont est porteuse la « communauté autochtone » contemporaine en

1. La Ley de Comunidades Nativas y de Promoción Agropecuaria de las Regiones de Selva y Ceja de Selva (loi n ${ }^{\circ} 20653$ ) est promulgée en 1974, dans la dynamique de la réforme agraire impulsée par le régime militaire de Juan Velasco Alvarado. Ce texte sera modifié en 1977 (loi $\mathrm{n}^{\circ}$ 22175), à l'initiative du gouvernement du général Morales Bermúdez. Si cette loi garantie théoriquement l'inaliénabilité des terres communales - du moins jusqu'à la Constitution de 1993 -, l'État central, profondément jacobin, reste propriétaire de l'eau, de la faune et du sous-sol: il cède en usage le sol et les ressources forestières aux communautés. Il se réserve donc le droit d'octroyer des parcelles à des entreprises extractives, comme les sociétés pétrolières, en dépit des conséquences de ces activités pour l'environnement et pour la santé des populations. Pour les gouvernements de l'époque, l'inscription de ces collectifs dans un maillage administratif complexe, l'élection de représentants chargés d'incarner localement l'État, dont la comunidad nativa n'est finalement qu'une sorte d'excroissance, sont autant de façons d'asseoir la légitimité des institutions publiques dans le quotidien des Amérindiens. 
Amazonie, instrument d'une souveraineté amérindienne autant que dispositif de colonisation sur la longue durée.

Aussi, si l'introduction du régime de la comunidad nativa représente, pour beaucoup d'Amérindiens, une promesse d'autonomie retrouvée après les périodes les plus sombres de l'exploitation du caoutchouc naturel des basses terres péruviennes, la façon dont les communiers ${ }^{2}$ objectivent leur propre processus de regroupement trahit fréquemment, sous l'influence du discours missionnaire, une moralisation des concepts liés à l'idée de « communauté ». Les ecclésiastiques des différentes obédiences - tant catholiques que protestantes - ont en effet largement défendu les vertus présumées de la corésidence communale. Après les affres de la colonisation, celle-ci devait assurer la recomposition culturelle des groupes autour d'un collectivisme qui leur était supposé «naturel $»^{3}$. Doublée en outre d'une politique de scolarisation systématique, la vie communale devait mettre les Amérindiens sur la voie de la « civilisation », promettant un dépassement substantiel de la « sauvagerie » et de l'« ignorance » des temps anciens. De sorte que, pour reprendre les mots de Stoll et Folhes (2014, p. 83-84), « la formation du village suggère, dès ses débuts, une reclassification sociale et une évaluation morale de l'espace et de ses occupants $»^{4}$.

C'est le processus de formation de Palotoa-Teparo, petite communauté administrative matsigenka du département du Madre de Dios, qui sera étudiée

2. Au Pérou, les membres inscrits dans les registres communaux sont désignés par le terme de comuneros, difficilement traduisible en français. Nous proposons d'utiliser le terme de « communier », employé dans la version française de Customs in common, d'Edward P. Thompson (2015). Traduit de l'anglais commoners, les communiers représentent « l'ensemble des individus qui avaient, dans les campagnes, le droit d'utiliser les biens communaux et qui étaient, de ce fait, des acteurs clés du monde rural, défenseurs de la solidarité communautaire » (Boutier et Virmani 2015, p. 37). Cette terminologie semble appropriée à notre cas d'étude, d'autant qu'elle permet de rappeler implicitement l'implication des missionnaires, catholiques et protestants, dans les regroupements des Amérindiens au sein de « communautés ».

3. Il s'agissait de créer localement de nouveaux pôles d'identification permettant de dépasser une « indistinction » de la masse indigène que les ecclésiastiques déploraient, et qui avait été encouragée selon eux par l'exploitation coloniale des basses terres.

4. Stoll et Folhes font ici référence aux communautés riveraines de Santarém, en Amazonie brésilienne (État du Pará), qui ont été formées dans les années 1960-1970 à l'initiative de missionnaires catholiques proches de la théorie de la libération. Les auteurs soulignent que si ce mode de résidence a d'abord été l'objet de critiques voire de rejets de la part des Amérindiens, ces derniers se sont peu à peu mobilisés pour « s'approprier un territoire idéel et symbolique qu'ils avaient tous contribué à forger et qu'ils considéraient désormais leur: l'idée d'une " communauté » solidaire et un ensemble de bénéfices incarnés dans la vila, principalement l'accès aux droits citoyens (école, église, infrastructures, affiliation administrative au syndicat, à la commune, inscription sur les listes électorales, etc.), mais aussi et surtout la participation à une forme de sociabilité morale qui est valorisée par opposition aux modes de vie des temps anciens où les riverains vivaient comme des "bêtes dans la forêt" » (Stoll et Folhes 2014, p. 96). 
au premier chef. Ce processus sera éclairé par quelques témoignages complémentaires recueillis dans le bas Urubamba (région de Cusco), où réside la majorité de la population matsigenka $\mathrm{a}^{5}$. On reviendra d'abord sur l'histoire du regroupement de Palotoa-Teparo, le sens que les Matsigenka lui donnent, avant d'explorer l'exercice contemporain du politique dans le cadre imposé par l'État.

Ce texte repose sur deux hypothèses de travail, élaborées au fur et à mesure de nos enquêtes de terrain. La première d'entre elle est que c'est en assumant leur identité politique de communier, c'est-à-dire d'individu inscrit dans les registres publics, qu'une partie des membres de cette ethnie se construit aujourd'hui comme « groupe » et qu'elle tente d'assurer l'interface avec l'État, ses administrations, voire avec le reste de la société nationale. Cela implique que, comme le montre l'enquête, les Matsigenka regroupés en « communautés ${ }^{6}$ » reprennent à leur compte le répertoire administratif imposé, qu'ils s'approprient son lexique et ses outils de manière à construire une représentativité politique qui leur est propre. De la sorte, ce sont des ramifications de l'État que les communiers ne cessent de reproduire, tout en exerçant, mutatis mutandis, une forme d'autonomie politique sur le territoire qui leur a été reconnue.

Ce point nous amène à notre deuxième hypothèse, selon laquelle l'activité politique des communiers consisterait, pour une large part, à envisager la « communauté » qu'ils administrent à l'image de l'État, en tant que celui-ci délègue les schèmes et dispositifs à partir desquels ils vont adosser leurs propres pratiques. Dans ce rapport de subsidiarité ainsi entretenu à l'égard de la puissance étatique, on ne cesse d'être surpris par le fait que l'hégémonie semble tout à la fois détournée et subrepticement reproduite. C'est une ambigüité comparable que Thomas Abercombrie mettait en lumière dans un article consacré aux liens entre ethnogenèse et domination coloniale dans les Andes boliviennes:

Ma thèse est que les unités ethniques modernes telles que nous les connaissons aujourd'hui, comme les Macha ou les K'ulta, sont issues de la prise en main par leurs ancêtres des stratégies administratives imposées, comme la réduction et

5. Les Matsigenka se répartissent historiquement entre les départements de Cusco et celui du Madre de Dios. Parmi les 12000 personnes qui composent cette ethnie parlant une langue la famille arawak, près de 80-85\% d'entre elles se trouve dans le bassin du bas Urubamba (Cusco), essentiellement entre le río Mishagua et la ville de Quillabamba. Les Matsigenka du Madre de Dios occupent pour leur part le haut Madre de Dios, le río Manu ainsi que ses affluents. Les Matsigenka seraient arrivés dans le bassin du haut Madre de Dios au début $\mathrm{du} \mathrm{XIX}^{\mathrm{e}}$ siècle, où ils établirent des relations avec les populations harakmbut de la région, relations marquées par des échanges et des conflits guerriers (Shepard et Izquierdo 2003).

6. Pour éviter les confusions entre les formes de socialité autochtones précoloniales et celles qui sont directement suscitées par les regroupements communaux contemporains, nous utiliserons systématiquement des guillemets pour parler de « communauté », ou bien nous préciserons qu'il s'agit de communautés administratives, autrement appelées « communautés natives » (comunidades nativas) en Amazonie péruvienne. 
Appropriation de la bureaucratie et expérience de la «communauté» chez les Matsigenka

les institutions de doctrine, pour reconstruire un système d'articulation qui servait autant leurs propres fins que celles de leurs dominateurs. [...] Dans les sociétés coloniales, la conquête n'est pas un événement singulier. La confrontation de systèmes culturels distincts est régulièrement recréée sur des formes rituelles en tant que locus de leur articulation historique. Elle préserve les formes de l'intervention coloniale tout en produisant les individus qui définissent les termes de l'articulation entre les ordres locaux et globaux. (Abercombrie 1990, p. 99)

Le cas de Palotoa-Teparo nous donne à voir, précisément, cette articulation paradoxale selon laquelle le modèle communal est, pour les Matsigenka, à la fois un instrument effectif d'émancipation, un dispositif acculturant autant qu'un générateur d'ethnicité.

\section{Palotoa-Teparo: genèse d'une communauté administrative}

Palotoa-Teparo est une petite communauté administrative du Madre de Dios dont la création a été encouragée par les missionnaires dominicains, mais mise en œuvre par un métis qui s'est peu à peu affranchi des catholiques. Située aujourd'hui dans la zone dite d' " amortissement » (amortiguamento) du parc national du Manu, Palotoa-Teparo réunit près de 130 personnes, toutes de langue matsigenka. On compte actuellement 48 adultes inscrits dans le registre communal. Installé sur la rive gauche de la rivière Shinkiveni, la « rivière du maïs », le regroupement fut formellement reconnu comme «communauté autochtone » (comunidad nativa) en 1987 et obtint son titre de propriété collective en 1990. Le territoire communal compte aujourd'hui plus de 6000 hectares ${ }^{7}$.

La présence missionnaire dans la zone remonte au début du $\mathrm{Xx}^{\mathrm{e}}$ siècle. Délégué en 1902 par l'ordre dominicain d'Espagne ${ }^{8}$, le père Zubierta a d'abord fondé une mission dans la vallée de Kosñipata, dans les contreforts des Andes, qui devint un lieu stratégique pour le projet d'évangélisation des Amérindiens des basses terres. Partant, Zubierta mandata les pères Osende et Pío Aza pour installer une mission à la confluence des fleuves Manu et Madre de Dios. Avec l'aide de deux colons locaux - Don Bernardino Pérez et Don Antonio Rengifo -, ils établirent la mission de San Luis del Manu en 1908, où se concentra l'essentiel de l'activité dominicaine après la fermeture de l'établissement de Kosñipata (Egido 2006, p. 42).

7. Pour l'octroi de son titre de propriété, la communauté administrative de Palotoa-Teparo a reçu initialement l'appui régulier d'une ONG dénommée Centre de développement de l'indigène amazonien (CEDIA), qui s'est peu à peu retirée au cours des années 2000, avant d'y cesser complètement ses activités à partir de 2010 .

8. L'Église catholique a distingué l'Amazonie péruvienne en trois régions (nord, centre et sud), confiant chacune d'entre elle à ses différents ordres - jésuites, franciscains et dominicains respectivement. Un recueil de textes produits par les dominicains et consacré aux Matsigenka a été récemment édité par Alonso Ordieres (2006). 
Plus en aval sur le haut Madre de Dios, un autre ecclésiastique, le père Vicente de Cenitagoya, tenta ensuite plusieurs incursions le long de la rivière Shinkiveni (ou Pantiacolla), où se situe actuellement la communauté administrative de Palotoa-Teparo, dans le but de fonder une nouvelle mission. Fuyant l'époque trouble de l'exploitation du caoutchouc ${ }^{9}$, plusieurs groupes s'y étaient en effet réfugiés. Rejetée par les Matsigenka, l'initiative missionnaire resta longtemps un échec. Ce n'est qu'en juillet 1921 que fut créée la mission de Nuestra Señora del Rosario de Pantiacolla, qui fut fermée en 1926 après la mort accidentelle de plusieurs individus. En 1958 enfin, après de nombreuses tentatives visant à rassembler les groupes harakmbut, l'actuelle mission de San Gabriel de Shintuya est finalement créée, à quelques kilomètres sur l'autre rive du Madre de Dios (ibid., p. 43-45).

Il faut par ailleurs souligner qu'à partir des années 1950, les missionnaires évangéliques de l'Instituto Lingüistico de $\operatorname{Verano}^{10}$ (Institut linguistique d'été, ILV) firent parallèlement leur entrée dans la région. Ils occupèrent l'aval du fleuve désormais délaissé par les dominicains, centrés comme on l'a vu sur le haut Madre de Dios. Ils regroupèrent plusieurs familles au sein de deux communautés administratives relativement importantes: Yomybato et Tayakome. Les évangéliques s'appuyèrent pour cela sur des Amérindiens ou des métis pouvant faire office de rabatteurs auprès des Matsigenka (Shepard et Izquierdo 2003). L'un d'entre eux, Vitaliano Cabrera, décédé dans les années 2000, fut l'un des fondateurs de Palotoa-Teparo.

Selon Guillermo Cabrera, fils de Vitaliano, son père était un métis né à Camisea, dans le bas Urubamba, d'une mère matsigenka et d'un père d'origine andine issu de Quillabamba. Présent à Tayakome au début des années 1970, l'anthropologue André-Marcel d'Ans (1981) mentionne le rôle de Cabrera,

9. La colonisation du Madre de Dios prend notamment son essor à la fin du XIX $x^{\mathrm{e}}$ siècle, lorsque l'entrepreneur Carlos Fermín Fitzcarrald parvient à relier la rivière Mishagua, un affluent de l'Urubamba et du bassin de l'Ucayali, à la rivière du Manu, précipitant la région dans le commerce du caoutchouc naturel. Accompagné de mercenaires lourdement armés, Fitzcarrald fera massacrer plusieurs milliers d'Amérindiens qui s'étaient rassemblés pour repousser les entreprises du cauchero (Casevitz 1969). Sur la violence de l'extraction du caoutchouc dans le Madre de Dios, voir également Piel (1980).

10. L'ILV est une émanation du Wycliffe Bible Translator (WBT), une organisation missionnaire évangélique nord-américaine fondée en 1942 par Guillermo Cameron Townsend (1896-1982). Le WBT s'était donné pour mission de traduire le Nouveau Testament dans toutes les langues du monde, et en particulier dans les langues autochtones, dans le but de les évangéliser. Sous la dénomination faussement académique d'ILV, le WBT s'est installé dans une quarantaine de pays du monde, traduisant leur Bible dans plus de 360 langues - dont 45 au Pérou. Sur l'entreprise d'évangélisation de l'ILV en Amazonie péruvienne, qui a parfois été qualifié d' " ethnocide » en raison de l'idéologie réactionnaire qui animait ses membres, voir en particulier d'Ans 1981; Hvalkof et Aaby 1981; Moore 1984. 
qu'il prénomme à cette époque « Italiano ${ }^{11}$. Il décrit un homme « intelligent », faisant preuve d'une grande éloquence et familier des grandes villes du sud du pays (Arequipa, Puno, Cusco). Visiblement doté d'une importante capacité d'influence sur ses pairs, Vitaliano Cabrera fut d'abord envoyé à Tayakome, où il commença à rassembler les familles dispersées le long du fleuve et de ses affluents pour le compte de l'ILV:

Vitaliano a regroupé les gens à Tayakome, il les a ramenés des affluents. Il leur apportait des haches, il leur apportait des machettes, des couteaux. Les linguistes donnaient tout cela à mon père et lui disaient: « Tu donnes ça à ceux qui vivent sur les affluents. Les enfants tu les ramènes ici pour qu'ils apprennent [à l'école]. » (Guillermo Cabrera, 29/08/2014, traduction de l'auteur)

Selon le récit de d'Ans (ibid.), Tayakome regroupait en 1971 plus de 200 Matsigenka. Les missionnaires dégagèrent pour cela un vaste espace de forêt et bâtirent une école en son centre. Autour de l'établissement étaient organisées des « rues » le long desquelles étaient disposées les familles. Avec la formation de la réserve naturelle protégée du Manu, en mai 1973, les autorités péruviennes tentèrent d'expulser les missionnaires et les Amérindiens. L'ILV incita alors les Matsigenka à se rabattre dans le bas Urubamba (région de Cusco), ce qui donnera naissance à la communauté administrative de Segakiato. Une partie des Matsigenka parvint néanmoins à rester à Tayakome, mais fut soumise à différentes interdictions liées à la règlementation du parc naturel qui sont en vigueur encore aujourd'hui, comme celle d'utiliser des armes à feu ou de faire commerce des ressources naturelles (Puygrenier Vargas 2007).

Cabrera se replia pour sa part sur la rivière Shinkiveni, où il jouera à nouveau un rôle de rabatteur, cette fois pour le compte des dominicains établis dans la mission de Shintuya. Une de ses filles, Sandy Cabrera, qui a été présidente de Palotoa-Teparo de 2011 à 2013, explique:

Mon père est celui qui les a fait se former, celui qui les a regroupés en communauté. Sans ça, nous aurions été au service des colons de Shintuya ${ }^{12}$, nous aurions été leurs esclaves. Mon père a appelé les familles qui étaient sur les affluents du Madre

11. Dans un texte des années 1980 dans lequel il revient sur sa présence à Tayakome, d'Ans (1981, p. 149) mentionne le rôle de Vitaliano Cabrera, dont il fait un portrait chaleureux. Un autre Matsigenka, dénommé Martín Vargas, aurait joué un rôle similaire lors de la formation de Tayakome.

12. La communauté administrative de Shintuya abrite aujourd'hui plus de 500 personnes, principalement des groupes de la famille linguistique harakmbut (des Arakbut et des Amarakaeri notamment). Un nombre important de colons d'origine andine y est également installé. Une piste relie Shintuya aux réseaux routiers qui permettent de se rendre dans la ville de Cusco. En outre, la mission catholique occupe toujours une position centrale dans la vie de la « communauté ». Jusqu'en 2018, elle était administrée par le père Pedro Rey, un prêtre dominicain originaire d'Espagne qui continuait à visiter ponctuellement les différentes communautés administratives du haut Madre de Dios pour y organiser des offices. 
de Dios, et elles se sont regroupées, et de là a commencé la communauté. Il leur a expliqué: « Nous nous regroupons pour que nous ayons notre territoire. Une fois que nous aurons notre territoire on fera ce que l'on voudra. Si nous voulons faire un jardin, nous ferons un jardin. Si au contraire nous restons [vivre] auprès des colons, ils vont nous prendre tout ce que nous avons, ils nous feront travailler sans cesse », leur a-t-il dit. Elles [les familles] ont compris et elles se sont regroupées pour obtenir le titre [de propriété] de la communauté. Comme à ce moment-là elles ne savaient pas jusqu'où pouvait aller la frontière de la communauté, jusqu'où elles pouvaient faire leurs maisons, il y a eu des difficultés pour l'obtention du titre. Nous avons rapidement fait construire l'école en dur, en ciment. (Sandy Cabrera, 21/02/2015, traduction de l'auteur)

Comme le souligne ce témoignage, l'un des principaux arguments justifiant la création de la communauté administrative était d'échapper à une forme de servitude vis-à-vis des colons locaux. L'arrivée de la comunidad nativa représente alors une autonomie retrouvée après les périodes les plus hostiles de l'avancée du front pionnier dans la région ${ }^{13}$. Toutefois, le sésame qui sanctionne la formation de cet espace d'émancipation est l'obtention d'un titre formel de propriété reconnu par l'État, la stabilisation de la vie communale coïncidant ainsi avec l'apprivoisement progressif des exigences des institutions publiques dont dépend l'octroi de ce titre. Comme on le verra plus bas, les communiers de Palotoa-Teparo souffriront en particulier de la possibilité de délimiter de façon précise le territoire communal, ce qui sera à l'origine de plusieurs expulsions.

\section{Un long chemin vers l'autonomie}

Les conditions de création de Palotoa-Teparo expliquent probablement l'esprit d'indépendance que la plupart de ses membres revendiquent. Certes, l'initiative du regroupement fut encouragée par les prêtres dominicains et ce sont eux qui firent construire la première école. Il semble néanmoins que Vitaliano Cabrera, conformément à son tempérament de franc-tireur décrit par d'Ans (1981, p. 149-150), s'affranchit rapidement de la mission de Shintuya voisine pour défendre l'autonomie de la communauté administrative qu'il avait contribué à

13. Peter Gow décrit un découpage historique similaire dans le discours des Yine de l'Ucayali : «Dans la narration de l'histoire des peuples autochtones, la Comunidad Nativa marque leur libération de l'esclavage. La reconnaissance des droits fonciers des peuples autochtones définit la communauté autochtone comme un caserio legítimo, un "véritable village", et symbolise leur autonomie résidentielle vis-à-vis des patrons. Les autochtones considèrent la Comunidad Nativa comme l'opposé de l'hacienda, lorsqu'ils vivaient dans l'esclavage des patrons, et comme un résultat historique de cette période » (Gow 1991, p. 211, traduction de l'auteur). Dans un sens proche, José Pimenta (2015) souligne que les Asháninka de l'Amônia (Brésil) insistent quant à eux sur le passage du «temps des patrons » au « temps des droits ». 
rassembler ${ }^{14}$. À ce titre, Vitaliano Cabrera incarne de manière typique la figure du curaca, servant d'intermédiaire avec les missionnaires et tentant de soutirer d'eux un maximum d'avantages pour le groupe de familles auquel il est lié ${ }^{15}$.

Au cours des années 1970-1980 vraisemblablement, un premier regroupement de cinq à six familles matsigenka vivait à l'embouchure du Shinkibeni. La situation resta stable durant une dizaine d'années. De l'autre côté de la rive se trouvait déjà la communauté administrative de Shintuya, où les Harakmbut sont majoritaires et avec qui les Matsigenka entretiennent de vieilles querelles. Alors que les communiers les plus anciens évoquent une époque où les différends se réglaient à jets de flèches, l'introduction du régime communal semble avoir déplacé ces tensions dans le champ administratif. Au début des années 1990 en effet, les résidants de Shintuya font valoir un droit de propriété sur le territoire occupé par les Matsigenka. Á force de tractations, ils obtiennent le départ de la communauté administrative, obligée de migrer plus en amont sur le Shinkibeni, à l'embouchure d'un petit ruisseau, le Jerontoria. L'épisode se répète à nouveau en 2000, repoussant le regroupement de Palotoa-Teparo plus haut encore sur la rivière. Sandy Cabrera se rappelle :

Ils nous ont expulsés et nous ont dit: « Allez-vous-en sur le terrain qui vous est dû. » Mon père disait: « Ils nous ont rejetés, qu'allons-nous faire? » Nous sommes partis en amont et nous ne nous sommes pas rendu compte que nous étions toujours sur le territoire de Shintuya. Nous avons vécu là dix ans et à nouveau, ils nous ont expulsés, ils nous ont rejetés et, après ça, nous sommes venus ici. Mais là aussi nous avons construit rapidement une école en béton, en ciment. (Sandy Cabrera, $21 / 02 / 2015$, traduction de l'auteur)

L'événement est cette fois retranscrit dans les comptes rendus écrits que les communiers tiennent rigoureusement à chacune de leur assemblée mensuelle. Quelques semaines avant l'expulsion, on y fait état des repérages des lieux potentiels et des travaux de défrichements. Le procès-verbal de la réunion du 8 août 2000 mentionne un débat sur la façon dont doit s'organiser le déplacement. Faut-il reconstruire en priorité un nouveau local communal ou bien les structures de l'école primaire? « Ce point a suscité une ample discussion, et nous sommes parvenus à prioriser en premier lieu le local scolaire du Centre éducatif, préoccupation première des communiers et des familles » (Palotoa-Teparo, assemblée générale du 15/08/2000, traduction de l'auteur). Les communiers décident à cette occasion de solliciter du matériel à la municipalité du district.

14. Lors d'une discussion en octobre 2015, le père Pedro Rey Fernández, alors administrateur de la mission de Shintuya, décrivait - non sans amertume - Vitaliano Cabrera comme un homme autoritaire et défendant farouchement l'autonomie de Palotoa-Teparo.

15. Sur l'imposition missionnaire de la figure du curaca en Amazonie péruvienne, voir notamment Chaumeil (1990). Dans le cas spécifique des Matsigenka, voir également Rosengren (1987). 
La requête est l'objet d'un document écrit et une « délégation » est désignée pour aller le porter aux autorités. On y demande 60 sacs de ciment, 200 plaques de tôles ondulées, 60 gallons d'essence, des clous, etc.

L'un des éléments notables qui ressort de cet épisode est que l'école est l'une des entités à partir de laquelle la communauté administrative se reconstitue en cas de difficulté. Elle est à la fois le signe d'une assise concrète autour de laquelle s'établir et d'un développement continu de la communauté administrative. À nouveau, il faut invoquer ici l'influence du discours missionnaire, qui a largement insisté sur les vertus civilisatrices de la scolarisation, perçue comme un processus corollaire au rassemblement des Amérindiens au sein de " communautés $»^{16}$. Néanmoins, il ressort aujourd'hui du témoignage des communiers que la mise à l'école de leurs enfants, si elle contraste indubitablement avec les formes de transmissions familiales des savoirs et des pratiques, garantit la formation d'une génération rompue aux mécanismes de la négociation administrative, en particulier grâce à l'apprentissage de la lecture et de l'espagnol. L'établissement symbolise ainsi la consolidation progressive et continue du groupe, qui existe dorénavant sous cette forme politique et administrative spécifique ${ }^{17}$.

16. Aussi, l'importance de la scolarisation dans le processus de formation de Palotoa-Teparo apparaît-t-elle clairement dans le récit de Guillermo Cabrera: « Les familles venaient du haut du fleuve, elles vivaient le long des affluents. Vitaliano est allé les rencontrer et les a fait se regrouper ici pour construire une école, pour que les enfants apprennent. Pour qu'ils puissent lire certaines choses, pour que certains nous aident à négocier les [ventes de] bananes, le nécessaire. Alors Vitaliano en a regroupé quelques-uns, les autres sont restés là-haut. [...] Petit à petit les familles sont venues se rapprocher » (Guillermo Cabrera, 29/08/2014, traduction de l'auteur). Un autre témoignage, recueilli cette fois dans le bas Urubamba (communauté administrative de Nuevo Mundo, formée à l'initiative des missionnaires protestants), nous semble également particulièrement éloquent: «- [Pour les missionnaires de l'ILV], la communauté et l'école étaient l'unique solution pour protéger les gens. Cet enfant n'allait pas être vendu parce qu'il allait à l'école. Ou plutôt, il allait être vendu à l'école. Il y a eu un changement. Avant on vendait les enfants à un patron, et maintenant on vend l'enfant à l'éducation. Que l'enfant apprenne à faire quelque chose, et qu'il vive au sein de la communauté. Tandis qu'un enfant qui est vendu à un patron disparait, il se perd, il s'intègre dans la société métisse » (Angel Diaz, 06/06/2017, traduction de l'auteur).

17. Peter Gow $(1991,2001)$ souligne également la centralité de l'établissement scolaire au sein des communautés administratives yine de l'Ucayali : «L'école, comme institution, est clairement centrale pour les communautés autochtones. Chaque jour de l'année, l'école domine la vie quotidienne du village. [...] Même les jours où il n'y a pas classe, comme les dimanches ou les jours de fêtes, l'école est le centre des activités du village, que ce soit pour des services religieux ou pour danser. L'école est la partie la plus "civilisée" du village, en tant que lieu où le savoir civilisé est transmis et où l'édifice est construit sur une structure proche de celle de la ville » (Gow 1991, p. 247, traduction de l'auteur). L'étude que Laura Rival (1996, p. 271-272) consacre aux Huaorani d'Équateur insiste tout autant sur l'importance de l'établissement scolaire au sein des regroupements amérindiens. Toutefois, l'auteure insiste moins sur l'intégration de cette institution dans l'univers symbolique amérindien, que sur les bouleversements qu'elle suscite sur la morphologie des groupes. La scolarisation provoquerait en effet un « changement social structuré et structurant » 
Selon Guillermo Cabrera, la première école de Palotoa-Teparo aurait ouvert avec une douzaine d'enfants. À la différence des évangéliques de l'ILV - qui utilisaient les langues vernaculaires pour parfaire leur travail d'évangélisation -, les prêtres catholiques, à l'origine de ce premier établissement, y dispensaient leurs enseignements en espagnol exclusivement. Comme l'a souligné Sheila Aikman (2003, p. 78), le peu d'intérêt des dominicains pour les langues autochtones les amènera à recruter des enseignants venus des hautes terres, souvent quechuaphones. En déléguant sur place ces professeurs, les dominicains limitèrent de fait leur propre présence ${ }^{18}$, ménageant une marge d'autonomie des communautés administratives vis-à-vis de la mission, à l'instar de Palotoa-Teparo.

Comme on l'a vu, l'établissement scolaire et sa construction revêtent une dimension centrale dans la formation de la communauté administrative, reléguant finalement les activités prosélytes à un second plan. L'intensité du rayonnement de l'école, autour de laquelle se presse aujourd'hui l'essentiel de la vie communale de Palotoa-Teparo, contraste en effet avec l'activité de la petite chapelle de bois - le choix du matériau n'est pas anodin - qui jouxte les salles de classe. La première abrite nombre d'activités qui ne concernent pas simplement la classe (réunions politiques, fêtes, jeux, activités sportives), tandis que la seconde tombe à l'abandon, n'étant plus fréquentée que deux ou trois fois l'an par le prêtre dominicain de Shintuya. Au contraire, c'est de ciment armé que l'école est bâtie, emblème de modernité et de durabilité, comme les communiers ne manquent pas de le souligner. Enfin, et ce dernier point est fondamental, c'est l'école qui accueillera les assemblées mensuelles de Palotoa-Teparo, aucun local spécifique n'ayant été construit par la suite. De sorte que l'établissement scolaire s'apparente à un « lieu anthropologique », au sens donné par Marc Augé (1992) à ce terme, c'est-à-dire un espace auquel les individus s'identifient, où ils établissent des relations sociales durables et autour duquel ils construisent une histoire collective.

\section{Ce que la communauté administrative veut dire}

Si le bon fonctionnement de l'école est l'un des sujets de préoccupation majeurs des communiers, sinon une des façons d'assurer la (re)production

chez les Huaorani, qui tend à convertir les regroupements en « unités sociales durables ». La mise à l'école fait notamment expérimenter une nouvelle « façon de se vêtir ou de se procurer de la nourriture qui participe à la fabrication des gens "civilisés" et [qui] cesse, dès lors qu'il n'y a plus de professeur ». Rival note en effet que les villages qui perdent leur établissement tendent à se défaire.

18. Dans les années 1950, les écoles fondées par les missionnaires dominicains sont regroupées via un réseau scolaire appelé le Red Escolar de la Selva Sur Oriente Peruano (RESSOP, Réseau scolaire de l'Amazonie sud-orientale péruvienne), qui est reconnu par le ministère de l'Éducation péruvien en 1971. 
de la communauté administrative, il y a par conséquent un enjeu, autant pour les parents que pour les enseignants de Palotoa-Teparo, d'augmenter le nombre d'élèves inscrits dans l'établissement. Une des stratégies consiste à convaincre les familles qui vivent de façon encore très isolée en dehors du territoire communal, en amont de la rivière Shinkiveni, de venir s'établir dans la communauté administrative afin de peser démographiquement face aux colons andins qui se montrent souvent avides d'exploiter les ressources sylvicoles de la zone. Les communiers, qui entretiennent parfois des liens de parenté anciens avec ces familles, leur rendent fréquemment visite lors de sorties de pêche ou de chasse qui s'étendent sur plusieurs jours. En échange de leur rattachement à la communauté administrative, ils leur proposent de les aider à construire des maisons et leur garantissent des espaces de culture, voire des alliances. Cette stratégie convient également aux enseignants, pour qui le fait d'avoir un nombre conséquent d'élèves leur permet d'exiger auprès des autorités locales davantage de matériel scolaire, de soutien logistique, ou encore de capter une part plus importante des programmes d'aide alimentaire à l'attention des écoles rurales.

Ainsi, début 2017, après avoir essuyé plusieurs défections et avoir expulsé différentes personnes considérées comme trop peu impliquées dans le fonctionnement de la communauté administrative, Palotoa-Teparo accueille deux nouvelles familles venues de l'amont de la rivière, dont sept enfants en bas âge. Inquiétées par l'attaque d'un jaguar (matsontsori), ces familles ont été ramenées par un communier qui les a convaincues d'y trouver un espace de sécurité. Les autorités de la communauté administrative se sont aussitôt chargées de leur créer une existence légale, faisant le nécessaire pour leur octroyer des cartes nationales d'identité (DNI), processus long et complexe qui a impliqué de fréquents voyages à Cusco. Après avoir reçu des patronymes à consonance hispanophone, leurs enfants ont été inscrits à l'école, où ils ont appris leurs premiers mots d'espagnol ${ }^{19}$. L'initiative des communiers à l'égard de ces familles peut paraître ambiguë. Il y a d'abord un projet politique, qui est de résister aux pressions coloniales contemporaines en leur opposant une force démographique amérindienne plus importante. Mais, de manière plus indirecte, les communiers en viennent à perpétuer le projet missionnaire de regroupement et de mise à l'école des Amérindiens, autrement dit le projet de contrôle et de « civilisation ».

19. On peut imaginer l'étrangeté qu'a dû représenter, pour ces enfants, le fait de devoir se rendre quotidiennement dans l'espace clos de la salle de classe, où les conditions de transmission des savoirs sont fondées sur une stricte organisation de l'espace et du temps, sur la définition des rôles et des places de chacun, de même que sur le contrôle de la parole et du geste qui organisent l'ordre scolaire et en génèrent les diverses modalités (Marchive 2007). 
Ajoutons encore que ces personnes récemment intégrées à la communauté administrative ne participent que rarement aux assemblées communales mensuelles, aux dépens de la règle générale qui veut que les communiers soient soumis à une amende (multa) en cas d'absence. Comme nous l'expliquait Ruben Semperi, qui par deux fois a été porté à la présidence de Palotoa-Teparo, cette clémence s'explique notamment par leur ignorance du fonctionnement de la communauté administrative :

- Pourquoi est-ce que les gens récemment intégrés à la communauté ne viennentils pas systématiquement aux assemblées?

- Ils viennent du haut de la rivière, ils sont arrivés il n'y a pas longtemps. Ils ne connaissent pas encore les statuts de la communauté. Ils ne savent pas, nous ne pouvons pas leur dire « respectez les statuts ». Ils vivent en amont [de la rivière], ils ont leur maison, ils vont pêcher, ils sortent, ils collectent des fruits. Leur vie se résume à cela, rien de plus. Ils n'ont jamais été au courant de la façon dont s'organise une communauté. Qu'est-ce qu'ils savent des statuts, des lois? Ils ne savent rien! Donc on ne peut pas leur exiger d'aller aux travaux collectifs comme les autres personnes qui sont inscrites dans le registre des communiers. À eux, oui, on le leur exige. Mais aux autres, non, ce sont des communiers en « contact initial» qui sont en processus d'intégration dans la communauté. Nous ne pouvons pas les forcer [en leur disant]: «travaille comme nous ». Il vaut mieux qu'ils intègrent eux-mêmes le fonctionnement de la communauté. Par exemple Japon vient à des réunions, parfois il vient lors de travaux collectifs et, petit à petit, il s'intègre. Il n'y a pas seulement des travaux collectifs, il y a aussi des donations, par exemple dans la cantine communale, il y a parfois des vêtements. À lui aussi on lui distribue [ces biens] à égalité avec les autres. Donc, en échange de tout cela, il doit se rendre compte qu'il doit lui aussi participer à l'avenir [de la communauté administrative]. Le bénéfice ne revient pas seulement au communier mais à tout le monde, et par conséquent nous devons travailler tous ensemble. [...] Petit à petit, ils vont apprendre. Avant c'était le même problème dans la communauté. Lorsque je suis arrivé pour la première fois, personne ne savait ce qu'étaient les lois. Nous avons organisé la communauté, petit à petit. Certains vivaient loin, désunis, ils travaillaient dans leur coin. Le travail doit toujours se faire de manière commune, [il faut] des travaux mutuels, pour que la communauté puisse s'organiser. C'est comme cela que pense la communauté. Avant, personne ne respectait [les contraintes collectives]. Il n'y avait pas de travaux collectifs, il n'y avait pas d'organisation capable de réclamer devant les autorités, pour les besoins de la communauté. [Il ne s'agit pas] seulement d'être dans la communauté; mais d'aller dehors, de réclamer... Parce que l'État soutient tout le monde. [...] Les besoins de la communauté, il faut les [lui] réclamer. (Ruben Semperi, 24/11/2014, traduction de l'auteur)

Le témoignage de Ruben Semperi, que nous avons choisi de citer longuement, est très explicite quant au processus de construction de la communauté administrative. Il soulève le fait que l'intégration politique est, sur le plan individuel et collectif, le fruit d'une longue acclimatation. Ce point ne peut être pleinement compris qu'au regard des modalités d'occupation traditionnelles du territoire 
liées au principe de parenté, qui se caractérisent, comme cela est évoqué dans le témoignage, par la dispersion des groupes de résidence ${ }^{20}$. Aussi, l'ethos résidentiel privilégié par les Matsigenka est marqué par la fluidité et la discontinuité, tandis que le territoire (kipatsi) est théoriquement extensif et ne peut être circonscrit aux limites étroites d'un cadastre communal (Arias 2006). Il s'agit d'autant de conceptions de la territorialité et de la résidence contre lesquelles les leaders communaux sont désormais forcés de lutter, faisant de l'« unité » de la communauté administrative un idéal instable et toujours à reprendre ${ }^{21}$.

Notons toutefois que pour donner sens à ces obligations collectives inédites, on tend à exiger des nouveaux arrivants ce qui, traditionnellement, est demandé aux beaux-fils dans le cadre de leur service marital. À l'instar de ces derniers en effet, les futurs communiers entrent dans une période dite de « probation » au cours de laquelle ils doivent démontrer, parfois plusieurs années durant, leur engagement pour le collectif en s'investissant activement dans les tâches communes et les réunions - même si un délai d'adaptation est octroyé aux personnes particulièrement étrangères à la communalisation. Pour ceux qui arrivent effectivement dans la communauté administrative dans une position de futurs époux, il s'agit en quelque sorte d'une extension du service marital, dans la mesure où c'est par l'ensemble des communiers réunis en assemblée

20. Constitués généralement d'une quinzaine de personnes, les groupes résidentiels matsigenka ont traditionnellement pour origine un couple fondateur autour duquel gravitent leurs filles et leurs gendres. Le mode de résidence privilégié étant matri-uxorilocal, les jeunes hommes viennent s'établir sur le territoire de leurs beaux-parents, tandis que les femmes restent habituellement ancrées dans un territoire qui est le leur. Il n'est pas possible de détailler ici les modes d'occupation du territoire liés au système de parenté, sur lesquels France-Marie Renard-Casevitz a écrit des articles capitaux (1977, 1993, 2007). Par ailleurs, le travail de Renard-Casevitz bat en brèche l'idée que la société matsigenka serait politiquement et socialement atomisée, comme l'ont longtemps pensé les observateurs extérieurs, et particulièrement les missionnaires catholiques. Au contraire, de vastes réseaux d'alliances commerciaux et guerriers liaient non seulement les Matsigenka entre eux, mais également l'ensemble des groupes arawak de l'Amazonie du piémont andin. Ces alliances, plus ou moins éphémères, ont longtemps permis de repousser les avancées coloniales hors des basses terres, du moins jusqu'à l'intensification de l'exploitation du caoutchouc, à la fin du XIX ${ }^{\mathrm{e}}$ siècle.

21. Dans un article consacré aux Korowai de Nouvelle-Guinée, Rupert Stasch décrit les regroupements de cette population au sein de villages, après avoir privilégié un mode de résidence plus dispersé. L'auteur évoque une périodisation relativement similaire à celle qui est faite par les Matsigenka: « Ces villages contrastent fortement avec l'ancien système spatial de résidence dispersée, comprenant non seulement le nombre de personnes vivant ensemble dans un même lieu, mais également le style architectural, la permanence de l'emplacement et l'étendue de l'abattis de la végétation. Dans divers contextes de discours, les Korowai périodisent leur histoire en un "temps sans villages" (xampung-alin-alüp) et un "temps où les villages ont été construits" (xampung-dotel-alüp). La géographie des espaces contrastés est vécue comme une expérience historique et le temps de l'histoire comme une expérience géographique » (Stasch 2017, p. 442, traduction de 1'auteur). 
Appropriation de la bureaucratie et expérience de la «communauté» chez les Matsigenka

- et pas uniquement par leurs futurs beaux-parents - qu'ils seront potentiellement évalués et sollicités. Pour les non-gendres, cette mise à l'épreuve relève davantage d'un calque du schème traditionnel des obligations et des devoirs du service marital qui, dès lors, est effectué dans le cadre des institutions de la communauté administrative contemporaine.

Un autre élément qui ressort du témoignage de Ruben Semperi est l'importance de l'arsenal légal - ce qu'il nomme les statuts et les lois - qui contribue à faire tenir le collectif sous cette forme politico-administrative spécifique qu'est la comunidad nativa. Agglomérer les individus et communaliser les efforts et les bénéfices est alors une manière de réclamer davantage de biens auprès des institutions publiques. Toutefois, l'enquête indique que l'appropriation des catégories légales et administratives n'est pas réductible à une stratégie de négociation - ou de diplomatie - avec l'État et ses administrations. Ces catégories, nous semble-t-il, acquièrent en effet une autonomie propre à l'intérieur de la communauté administrative.

\section{La fabrique du droit communal}

Soulignons tout d'abord qu'un grand nombre de règles de vie, qui vont de la gestion du territoire et de ses ressources à l'incorporation de nouveaux membres, sont consignées dans des statuts communaux, que les communiers de Palotoa-Teparo qualifient parfois de « lois ». Ces statuts ont une base commune à l'ensemble des communautés administratives autochtones au Pérou, Andes et Amazonie confondues, à savoir des textes légaux comme le Code civil ou la loi des communautés natives. Ce sont ces statuts qui obligent par exemple les Amérindiens à organiser des assemblées mensuelles, à charge d'un comité directeur (Junta Directiva) élu tous les deux ans. Ce comité a ainsi un rôle institutionnel fondamental et la législation péruvienne en fait « l'organe de gouvernement, d'administration et de représentation légale de la communauté » (loi des communautés natives, chap. 4, art. 23, traduction de l'auteur). À Palotoa-Teparo, les statuts communaux comportent 70 articles. Ceux-ci ont été modifiés par deux fois dans le cadre d'assemblées communales.

La loi impose par ailleurs qu'un suivi des réunions soit réalisé de façon systématique via des procès-verbaux, autrement appelés Actes d'assemblées (Actas de asambleas), rédigés par un secrétaire dont c'est le rôle. Ces comptes rendus sont archivés dans des cahiers visés annuellement par un juge de paix, ce qui en fait des documents légaux. Nous avons pu longuement consulter les Actes de Palotoa-Teparo, qui sont tenus de manière systématique depuis mars 1997. Au fil de plus de 1000 pages d'une écriture souvent hésitante et aux formules ampoulées, regroupées en cinq cahiers de grand format, les communiers ont ainsi rendu compte du déroulement des assemblées mensuelles ordinaires 
(une fois le mois), et parfois extraordinaires, lorsqu'un événement impromptu nécessitait une réunion d'urgence. Ces Actes décrivent, souvent avec force détails, les propos des communiers, leurs prises de position, les débats qui les opposent. Sont également consignés les rapports (informes) que chacun fait concernant des tâches qu'il a accomplies durant le mois écoulé, les missives qu'une quelconque autorité publique locale a pu confier à l'attention de la communauté administrative. On y exprime enfin certaines requêtes, comme l'autorisation de couper un arbre, ou de l'aide pour certains travaux. Il est à noter que l'étude de ce corpus ne permet pas de découvrir d'emblée ce qui serait la véritable motivation de l'institution communale et de son assemblée. Au contraire, c'est en revenant sur le modus operandi d'un tel document, autrement dit sur les « opérations du droit » (Thomas 2011), que l'on peut saisir la façon dont l'institution est peu à peu produite ${ }^{22}$.

S'il n'est pas possible ici de décrire en détail les dynamiques internes des assemblées communales et le processus de ritualisation qui leur sont propres ${ }^{23}$, il faut néanmoins souligner que l'une des étapes cruciales de ces réunions est la lecture à voix haute des Actes de la réunion antérieure, exercice essentiel à la matérialisation du collectif et à son inscription dans une figure culturelle officielle: celle de la « communauté autochtone matsigenka », appartenant à tel district, à telle province et à tel département. Plus encore, ce que montre cette lecture publique, c'est que les cahiers d'Actes sont le lieu privilégié de fabrication d'une législation interne à la communauté. Au cours des débats qui émaillent les assemblées, ce sont en effet des propositions de type normatif qui apparaissent de manière itérative. Peut-on faire commerce du bois et du poisson? À quelles conditions et en quelles quantités? Quelles sont les conditions pour avoir le droit de s'absenter de la communauté? Quelles sont les sanctions pour ceux qui ne participent pas aux assemblées ou aux travaux communaux? Qui peut entrer dans le territoire communal?

De telles propositions sont alors discutées, rejetées ou acceptées par le vote, puis dûment consignées dans les cahiers d'Actes, ce qui contribue à enrichir la normativité prévue par les « statuts » officiels de la communauté administrative $^{24}$. Il faut, sans nul doute, souligner le rôle des ONG et des institutions

22. Comme l'indique l'historien Angelo Torre (2007, p. 103) à propos des actes communaux dans le Piémont italien médiéval, « il convient d'examiner, outre le discours que le document véhicule, la genèse du document lui-même ». Certains travaux de sociologie des institutions soulignent en ce sens que ce sont souvent les pratiques scripturaires, à savoir le travail de rédaction des documents administratifs, qui contribuent à « produire » l'institution. D'où l'intérêt d'analyser la littérature bureaucratique « en train de se faire » (Gayon 2016).

23. Un certain nombre de « rituels » administratifs tendent chaque fois à délimiter ces assemblées en tant que lieu politique distinct des affaires courantes (Colliaux 2019).

24. Dans le cas andin, Ingrid Hall (2015, p. 98) évoque le fait que durant les assemblées communales, « les orateurs déplorent régulièrement le fait que ces statuts n'envisagent 
publiques, qui encouragent, par des interventions ponctuelles mais soutenues, un tel quadrillage des activités quotidiennes. On peut prendre pour exemple les « plans de vie » (Espinosa de Rivero 2014) promus par ces organisations, et qui visent à délimiter géographiquement les activités de subsistance (chasse, pêche, agriculture), voire à définir des quotas de prélèvement - pratiques d'autant plus encouragées par la proximité du parc national du Manu tout proche. À Palotoa-Teparo par exemple, suite aux conseils d'une ONG de défense de l'environnement, on n'autorise la pêche au filet (plus dommageable écologiquement) qu'en certaines zones, on limite l'exploitation de bois à quelques parcelles, les activités touristiques - s'il y en a - à d'autres. Ces accords pris en assemblée sont largement respectés, et ceux qui s'en écartent sont sommés de se justifier (Colliaux 2019).

Les communiers ne cessent, par leurs propres pratiques, de reproduire ce processus de planification, travaillant activement à la création d'un registre de sanctions à appliquer le cas échéant. La production écrite devient alors un instrument de contrôle du territoire, des personnes et des activités qui s'y déroulent. Encastrant chaque fois davantage les rapports sociaux dans cette normativité interne, cette dynamique illustre par là même un souci d'exactitude et de prévisibilité des événements, où le passé est appréhendé à l'aune du présent légitimé ${ }^{25}$. Ainsi, la lecture publique des Actes, au début de chaque nouvelle assemblée, fait autant référence à un cadre normatif à respecter, qu'à un programme politico-administratif que l'on se propose de mettre en œuvre collectivement. Un jeune homme rencontré dans une autre communauté administrative matsigenka, celle de Kirigueti (bas Urubamba, région de Cusco) traduit bien cet aspect programmatique des Actes de réunion:

- Pourquoi les Actes sont-ils importants?

- C'est un document où l'on détaille les choses que l'on va faire et que l'on doit accomplir. Les choses qu'on doit réaliser et qu'on ne doit pas ignorer. Comme on dit, « ce qu'il s'est dit en réunion doit être écrit dans l'Acte ». (Harlin Burges, $31 / 07 / 2017$, traduction de l'auteur)

pas tous les cas; ce qui les oblige à prendre parti ». Mais l'auteur n'en tire pas les mêmes conséquences que nous, à savoir le déclenchement d'un processus jurisprudentiel interne à la communauté administrative.

25. Les analyses de Laura Baietto (2002) sur les communautés italiennes du XIII ${ }^{\mathrm{e}}$ siècle, et en particulier ce qui concerne l'écrit et la production d'une juridiction interne, méritent quelques rapprochements avec notre cas d'étude: " le système documentaire a désormais pour fonction d'organiser le présent et de prévoir le futur. Ainsi, les pratiques d'écritures sous forme de livres et de registres assument une fonction de contrôle de l'espace, c'est-àdire du territoire, et des personnes qui y résident, mais aussi du temps : sur le passé qui doit être décrit et interprété en fonction de la légitimation du présent, sur le présent qui doit être organisé et sur le futur qui peut être planifié » (Baietto 2002, p. 678, traduction de l'auteur). 
C'est également à Kirigueti que son jeune président, un ancien étudiant en anthropologie de l'université de Cusco, nous proposa d'apposer le sceau de la communauté administrative sur notre thèse, afin que nous puissions prouver de la validité de notre enquête auprès de notre établissement de rattachement. Cette anecdote témoigne, comme les précédentes, de l'efficacité symbolique de ces documents produits par ces communautés administratives. Cela contraste avec ce que l'on peut observer ailleurs en Amazonie amérindienne, où ce sont plutôt les documents produits à l'extérieur qui semblent dotés de performativité (Allard 2012; Gordillo 2006).

\section{Ciudadanos de la comunidad}

À partir du cas singulier du regroupement matsigenka de Palotoa-Teparo (département du Madre de Dios), cet article a voulu décrire les perspectives diverses qui se cristallisent autour de la notion de «communauté amérindienne » en Amazonie péruvienne. Tout se passe comme si, d'un côté, le modèle communal nous ramenait inexorablement à l'histoire coloniale du pays, et aux multiples missions « civilisatrices » que les populations autochtones ont dû affronter. Mais, d'un autre côté, il s'agit aussi - et désormais surtout - d'un puissant instrument d'émancipation, garantissant aux Amérindiens l'accès à un territoire propre, certes limité - tant physiquement que symboliquement -, mais effectif politiquement. En partant de cette ambivalence fondamentale de la « communauté administrative » contemporaine, nous avons alors interrogé la façon dont celle-ci vient organiser le commun politique amérindien à l'aide d'un répertoire administratif imposé par l'État, et au moyen duquel la socialité est objectivée dans les termes promus par les institutions publiques.

Ce que le cas de Palotoa-Teparo donne à voir, de manière exemplaire, c'est un travail d'intégration de ces catégories légales et administratives exogènes, au point de faire émerger, parmi les Matsigenka qui en font l'expérience, un mode spécifique de représentation et d'action politique. Or, cette singulière « conscience du droit » (Ewick et Silbey 2004), et la créativité politique locale qui en découle, ne sont pas dépourvues d'ambiguïté. Car dans l'exercice de cette souveraineté amérindienne, ce sont la bureaucratie et les structures administratives qui se trouvent automatiquement reproduites. Comment manœuvrer, dès lors, avec des schèmes pratiques et discursifs imposées par ces dernières, et qui ne cherchent, par nature, qu'à exprimer une « pensée d'État », davantage qu'une quelconque autonomie amérindienne? Comment appréhender ce rapport de subsidiarité entre la « communauté » que les communiers administrent et l'État péruvien?

Ce dilemme, et la façon dont les Matsigenka l'envisagent, ne sont peut-être jamais aussi apparents que lorsque que les communiers de Palotoa-Teparo se 
Appropriation de la bureaucratie et expérience de la «communauté» chez les Matsigenka

réunissent chaque mois dans une des salles de classe de l'école, espace qui, légalement, appartient à 1'État ${ }^{26}$. En investissant mensuellement l'institution scolaire et en produisant à cette occasion une ample documentation administrative qui a vocation à contrôler le territoire, les personnes et les activités qui s'y déroulent, ce sont certes des ramifications de l'État que la « communauté » amérindienne recrée activement, mais aussi qu'elle s'approprie, générant par là même les conditions de sa propre autonomie.

Une expression fréquemment entendue lors de nos séjours de terrain apporte un éclairage complémentaire. Les parents d'élèves nous soulignaient en effet que le passage par l'école communale permettait de former ce qu'ils appelaient des « citoyens de la communauté » (ciudadanos de la comunidad). En d'autres termes, plutôt qu'une communauté de citoyens semblablement intégrés à une nation moderne - autrement dit non-amérindienne -, c'est une communauté proprement autochtone, ici une communauté matsigenka, que l'établissement scolaire devait, aux yeux de ces parents, contribuer à produire. *

* Manuscrit reçu en juin 2019, accepté pour publication en novembre 2019.

Remerciements - Ce texte est tiré de notre thèse de doctorat en sociologie, soutenue en janvier 2019 à l'École des hautes études en sciences sociales (EHESS, Paris). Dans ce cadre, nous avons mené trois séjours de terrain entre 2014 et 2017, cumulant au total une dizaine de mois d'enquête. Ces séjours ont été financés grâce au concours du Labex Tepsis (EHESS), du Legs Lelong en anthropologie sociale (CNRS), ainsi que par une bourse de mobilité de l'Institut français d'études andines (Umifre 17 MEAE/ CNRS). Les dernières étapes de cette recherche ont été réalisées dans le cadre du projet AMAZ (ANR-17-CE41-0013). Je remercie chaleureusement Catherine Alès, Isabel Yaya McKenzie, l'équipe de rédaction du JSA ainsi que ses relecteurs anonymes pour leur révision attentive de ce travail et leurs précieux commentaires.

26. Le terrain qui abrite l'école - d'une surface de 4000 mètres carrés dans le cas de Palotoa-Teparo - est en effet une «donation » que la communauté administrative doit légalement concéder à l'État, étape nécessaire avant que celui-ci n'y construise l'établissement, et n'y délègue des enseignants. Ce procédé est l'objet d'une assemblée communale extraordinaire, puis, inévitablement, d'un procès-verbal dans les livres d'Actes de la communauté administrative. 


\section{Références citées}

ABercombrie Thomas

1990 «Ethnogenèse et domination coloniale », Journal de la Société des américanistes, 76, p. 95-104.

AIKMAN Sheila

2003 La educación indígena en Sudamérica interculturalidad y bilingüismo en Madre de dios, Perú, Instituto de Estudios Peruanos, Lima.

Allard Olivier

2012 «Bureaucratic anxiety: asymmetrical interactions and the role of documents in the Orinoco Delta (Venezuela) », Hau. Journal of Ethnographic Theory, 2 (2), p. 234-256.

Alonso Ordieres Rafael (éd.)

2006 La vida del pueblo matsiguenga. Aporte etnográfico de los misioneros dominicos al estudio de la cultura matsiguenga (1923-1978), Centro Cultural « Pio Aza »/Misioneros Dominicos, Lima.

Ans André-Marcel (d')

1981 «Encounter in Peru », in Soren Hvalkof et Peteer Aaby (dir.), Is god an American? An anthropological perspective on the missionary work of the summer Institute of Linguistics, IWGIA/Survival International, Copenhague, p. 145-162.

ARIAS Esteban

2006 « El tejido social matsigenka », in Martha Rojas Zolezzi et Cecilia Larrabure, La cultura ancestral matsigenka: respuestas a la modernidad del siglo XXI, Confederación de Nacionalidades Amazónicas del Perú, Lima, p. 22-43.

Augé Marc

1992 Non-lieux. Introduction à une anthropologie de la surmodernité, Le Seuil (La librairie du $\mathrm{xx}^{\mathrm{e}}$ siècle), Paris.

BAIETTO Laura

2002 «Elaborazione di sistemi documentari e trasformazioni politiche dei comuni piemontesi (sec. XIII): una relazione di circolarita », Societa e storia, 98, p. 654-679.

Boutier Jean et Arundhati Virmani

2015 «Présentation », in Edward P. Thompson, Les usages de la coutume. Traditions et résistances populaires en Angleterre. XVII ${ }^{e}-X I X^{e}$ siècle, EHESS/Gallimard/ Le Seuil (Hautes études), Paris, p. 9-44.

Chaumeil Jean-Pierre

1990 " "Les nouveaux chefs..." Pratiques politiques et organisations indigènes en Amazonie péruvienne », Problèmes d'Amérique latine, 96, p. 93-113.

Colliaux Raphaël

2019 «La "communauté" autochtone dans la longue durée. Perspectives comparatives au Pérou », cArgo. Revue internationale d'anthropologie culturelle \& sociale, 9, p. 66-87.

EGIDo Jesús M.

2006 Shintuya. Guía sanitaria de la zona, Centro Amazónico de Antropología y Aplicación Práctica, Lima. 
Appropriation de la bureaucratie et expérience de la «communauté» chez les Matsigenka

ESPINOSA DE Rivero Oscar

2014 «Los planes de vida y la política indígena en la Amazonía peruana », Anthropologica, 32 (32), p. 87-114.

Ewick Patricia et Susan SiLbey

2004 «La construction sociale de la légalité », Terrains \& travaux, 1 (6), p. 112-138.

Fioravanti-Molinie Antoinette

1978 «La communauté aujourd'hui », Annales ESC, 5-6, p. 1182-1196.

GAYON Vincent

2016 «Écrire, prescrire, proscrire. Notes pour une sociogénétique de l'écrit bureaucratique ", Actes de la recherche en sciences sociales, 3 (213), p. 84-103.

Gordillo Gastón

2006 « The crucible of citizenship: id-paper fetishism in the Argentinean Chaco », American Ethnologist, 33 (2), p. 162-176.

Gow Peter

1991 Of mixed blood. Kinship and history in Peruvian Amazonia, Clarendon Press, Oxford.

2001 An Amazonian myth and its history, Oxford University Press, Oxford.

HaLl Ingrid

2015 « Parole et hiérarchie dans les Andes du Sud du Pérou », Autrepart, 1 (73), p. 89-103.

Herzog Tamar

2007 «Terres et déserts, société et sauvagerie. De la communauté en Amérique et en Castille à l'époque moderne », Annales HSS, 3, p. 507-538.

Hvalkof Søren et Peter Aaby

1981 «Introducing god in the devil's paradise », in Søren Hvalkof et Peter Aaby (dir.), Is god an American? An anthropological perspective on the missionary work of the summer Institute of Linguistics, IWGIA/Survival International, Copenhague, p. 9-16.

Marchive Alain

2007 «Le rituel, la règle et les savoirs. Ethnographie de l'ordre scolaire à l'école primaire », Ethnologie française, 37 (4), p. 597-604.

Moore Thomas

1984 «El ILV y una "Tribu recién encontrada": la experiencia amarakaeri », América Indígena, 44 (1), p. 25-48.

PiEL Jean

1980 «Le caoutchouc, la Winchester et l'Empire », Revue française d'histoire d'outre-mer, 67 (248-249), p. 227-252.

Pimenta José

2015 «Du "temps des patrons" au "temps des droits": conflits interethniques et transformations politiques chez les Ashaninka d'Amazonie brésilienne », Journal de la Société des américanistes, 101 (1-2), p. 269-294.

Puygrenier Vargas Fany

2007 « Jardin interdit au cœur de l'Éden. Conflits d'intérêts dans le Parc national du Manu (Pérou) », Bulletin de l'Institut français d'études andines, 36 (3), p. 407-426. 
RENARD-CASEvitz France-Marie

1977 «Du proche au loin. Étude du fonctionnement des systèmes de la parenté et de l'alliance matsiguenga », Actes du $42^{e}$ Congrès international des américanistes, 2, p. 121-140.

1993 «Guerriers du sel, sauniers de la paix », L’Homme, 33 (126-128), p. 25-43.

2007 «"De l'égalité des deux sexes" au fondement de l'imaginaire social chez les Matsiguenga (Amazonie péruvienne) », in Nicole-Claude Mathieu (dir.), Une maison sans fille est une maison morte. La personne et le genre en sociétés matrilinéaires et/ou uxorilocales, Éditions de la Maison des sciences de l'homme, Paris, p. 155-182.

Rival Laura

1996 Hijos del sol, padres del jaguar. Los Waorani de ayer y hoy, Abya Yala, Quito. ROSENGREN Dan

1987 In the eyes of the Beholder. Leadership and the social construction of power and dominance among the Matsigenka of the Peruvian Amazon, Göteborgs Ethnografiska Museum, Göteborg.

ShePARd Glenn H. Jr. et Carolina IzQuierdo

2003 «Los Matsiguenka de Madre de Dios y del Parque Nacional del Manu », in Beatriz Huertas Castillo et Alfredo García Altamirano (dir.), Los pueblos indígenas de Madre de Dios. Historia, etnografia y coyuntura, FENAMAD/ IWGIA, Lima, p. 111-126.

STASCH Rupert

2017 «Afterword: village space and the experience of difference and hierarchy between normative orders ", Critique of Anthropology, 37 (4), p. 440-456.

Stoll Emilie et Ricardo Theophilo FolHes

2014 «La (dés)illusion communautaire. De l'ambivalence de la notion de "communauté" en Amazonie brésilienne », Journal de la Société des américanistes, 100 (2), p. 73-103.

Thomas Yan

2011 Les Opérations du droit, EHESS/Gallimard/Le Seuil (Hautes Études), Paris. ThOMPson Edward P.

2015 Les usages de la coutume. Traditions et résistances populaires en Angleterre. $X V I I^{e}-X I X^{e}$ siècle, EHESS/Gallimard/Le Seuil (Hautes études), Paris.

TORRe Angelo

2007 «"Faire communauté". Confréries et localité dans une vallée du Piémont (XVII ${ }^{\mathrm{e}}$ XVIII ${ }^{\mathrm{e}}$ siècle) », Annales HSS, 62 (1), p. 101-135.

ZAGALSKY Paula

2009 «El concepto de "comunidad" en su dimensión espacial. Una historización de su semántica en el contexto colonial andino (siglos XVI-XVII) », Revista Andina, 48, p. 57-90. 